\title{
Temporal Dependence in Uncoupling of Blood Volume and Oxygenation during Interictal Epileptiform Events in Rat Neocortex
}

\author{
Minah Suh, Sonya Bahar, Ashesh D. Mehta, and Theodore H. Schwartz \\ Department of Neurological Surgery, Weill Medical College of Cornell University, New York, New York 10021
}

\begin{abstract}
We investigated the dynamic spatiotemporal relationships of cerebral blood volume (CBV), deoxygenated hemoglobin (Hbr), and light scatter (LS) associated with interictal epileptiform events with multiwavelength optical recording of intrinsic signals and simultaneous field potential recording. Interictal spikes (IISs) were induced with iontophoresis of bicuculline methiodide in rat neocortex. Intrinsic signal changes appeared as early as $100 \mathrm{msec}$ after the IIS at all wavelengths and could be appreciated after only a single IIS. Initially, the largest signal arose from a focal increase in deoxygenation, which lasted for $\sim 2$ sec, consistent with an "initial dip." An equally early focal increase in CBV had a smaller amplitude than the Hbr signal until $>2$ sec after the IIS, when its amplitude surpassed that of the Hbr signal but also spread to a larger, less focal area. The most spatially restricted and smallest amplitude signal was produced by LS. A later hyperoxygenation, or increase in blood oxygenation level-dependent signal, was often seen in the draining veins but inconsistently seen in the IIS focus. An inverted optical signal was recorded at all wavelengths from multiple regions in the surrounding cortex within 100 msec of the IIS. We therefore conclude that the IIS induces a rapid increase in metabolic demand, which cannot be met by a rapid, initially focal but small increase in CBV that results in a prolonged increase in $\mathrm{Hbr}$ (epileptic dip in oxygenated hemoglobin). The inverted optical signal in the surround arises from a decrease in $\mathrm{CBV}$ and a decrease in $\mathrm{Hbr}$, likely resulting from a combination of shunting of $\mathrm{CBV}$ to the focus and decreased metabolic demand resulting from decreased neuronal activity, consistent with "surround inhibition."
\end{abstract}

Key words: bicuculline; epilepsy; neocortex; rat; interictal spike; optical imaging; intrinsic signal; hemoglobin; initial dip; cerebral blood volume; BOLD; fMRI

\section{Introduction}

Interictal spikes (IISs) recorded from epileptic brain represent a population of hyperexcitable neurons (Schwartzkroin and Wyler, 1980), which are usually contiguous with the ictal onset zone, the area of the brain from which seizures arise (Engel, 1990; de Curtis and Avanzini, 2001). Because of the infrequency of ictal events, the IIS is often used as a marker for epileptic cortex, particularly by clinicians in preparation for surgical therapy (Wyllie et al., 1987; Ojemann, 1993). Recently, perfusion-based imaging techniques such as positron emission tomography (PET), single-photon emission computed tomography (SPECT), and functional magnetic resonance imaging (fMRI), which rely on the coupling between neuronal activity, cerebral blood flow (CBF), and hemoglobin oxygenation, have recently become widely used in the clinical evaluation of patients with epilepsy to localize the IIS (Duncan, 1997; Lemieux et al., 2001a,b; Benar et al., 2002).

Received March 26, 2004; revised 0ct. 1, 2004; accepted 0ct. 1, 2004.

T.H.S. was supported by the Epilepsy Foundation Junior Investigator Award, American Association of Neurological Surgeons Young Clinician Investigator Award, Citizens United for Research in Epilepsy, and by National Institute of Neurological Disorders and Stroke Grant K08 NS43799. We thank Koon Ho (Danny) Wong for technical assistance and Dr. Jonathan Victor for help with this manuscript.

Correspondence should be addressed to Dr. Minah Suh, Department of Neurological Surgery, Weill Medical College of Cornell University, 525 East 68th Street, Box 99, New York, NY 10021. E-mail: mis2032@med.cornell.edu.

DOI:10.1523/JNEUROSCI.2823-04.2005

Copyright $\odot 2005$ Society for Neuroscience $\quad 0270-6474 / 05 / 250068-10 \$ 15.00 / 0$
The basic premise behind these techniques is that neuronal activity induces an increase in cerebral metabolic rate $\left(\mathrm{CMRO}_{2}\right)$, which in turn causes an increase in $\mathrm{CBF}$ and cerebral blood volume (CBV) as blood vessels dilate to bring oxygenated hemoglobin $\left(\mathrm{HBO}_{2}\right)$ to the area (Roy and Sherrington, 1890). Because the increase in CBF brings an overabundance of $\mathrm{HBO}_{2}$, there is a focal but slightly delayed $(>2 \mathrm{sec}$ ) alteration in the paramagnetic properties of hemoglobin, which can be imaged with fMRI (Fox and Raichle, 1986; Ogawa et al., 1990). This "uncoupling" is often referred to as the blood oxygenation level-dependent (BOLD) effect (Ogawa et al., 1990). In addition, several investigators have described a more rapid increase in deoxygenated hemoglobin ( $\mathrm{Hbr}$ ) after increases in $\mathrm{CMRO}_{2}$ that precede any change in $\mathrm{CBF}$ or BOLD signal (Malonek and Grinvald, 1996; Vanzetta and Grinvald, 1999; Mayhew et al., 2000; Sheth et al., 2003). This "initial dip" in hemoglobin oxygenation represents another type of uncoupling, which is quite controversial, because several laboratories have disputed its existence (Frannson et al., 1998; Mandeville et al., 1999a,b; Silva et al., 2000; Lindauer et al., 2001; Sheth et al., 2004). Because a complimentary reduction in $\mathrm{HBO}_{2}$ is rarely seen during the initial dip, some investigators have also predicted the existence of a rapid, highly localized increase in $\mathrm{CBV}$ within the first $2 \mathrm{sec}$ after neuronal activation (Kuschinsky and Paulson, 1992; Malonek and Grinvald, 1996). However, all data on the latency and spatial specificity of the changes in CBF, 
$\mathrm{CBV}, \mathrm{HBO}_{2}$, and $\mathrm{Hbr}$ after neuronal activity comes from studies involving normal sensory activation either in visual or somatosensory cortex (Frostig et al., 1990; Malonek and Grinvald, 1996; Vanzetta et al., 1999; Sheth et al., 2003, 2004). Little data exists on abnormal physiologic events such as epilepsy.

Previous investigations into the relationship between epileptiform events and perfusion or metabolism have demonstrated contradictory results in both animals and humans using autoradiography, PET, and fMRI, techniques with limited temporal and spatial resolution. Although an increase in perfusion is universally demonstrated, some studies find that perfusion oversupplies metabolism (Lemieux et al., 2001a,b; Benar et al., 2002; Nersesyan et al., 2004; Tenney et al., 2004), whereas others demonstrate the opposite, namely inadequate perfusion to meet metabolic demand (Ingvar and Siesjo, 1983; Tanaka et al., 1990; Kreisman et al., 1991; Pereira de Vasconcelos et al., 2002). We chose to investigate the relationship between perfusion, hemoglobin oxygenation, and epileptiform activity using optical recording of intrinsic signals (ORIS), which offers the highest combined spatial and temporal resolution of any technique (Schwartz et al., 2004). Depending on the wavelength of incident light, ORIS can provide information about CBV, Hbr, and light scatter (LS) with a temporal resolution of $\sim 100 \mathrm{msec}$ and a spatial resolution of $<200$ $\mu \mathrm{m}$ (Bonhoeffer and Grinvald, 1996; Granquist-Fraser et al., 2003; Schwartz et al., 2003) as well as an ability to sample large areas of neocortex simultaneously. Previous in vivo studies of epilepsy using ORIS have been performed at wavelengths $>650$ $\mathrm{nm}$, which is most sensitive to LS rather than perfusion or oxygenation (Haglund et al., 1992; Chen et al., 2000; Schwartz and Bonhoeffer, 2001; Schwartz, 2003). Hence, there is no data about the relationship between CBV and hemoglobin oxygenation after epileptiform events with the high temporal and spatial resolution provided by ORIS.

\section{Materials and Methods}

Animal preparation. Adult male Sprague Dawley rats $(n=10 ; 250-375$ $\mathrm{gm})$ were initially anesthetized with intraperitoneal ketamine $(90 \mathrm{mg} / \mathrm{kg})$ and xylazine $(4.0 \mathrm{mg} / \mathrm{kg})$ and then sustained with urethane $(1.25 \mathrm{gm} / \mathrm{kg})$, decadron $(0.17 \mathrm{mg} / \mathrm{kg})$, mannitol $(0.83 \mathrm{gm} / \mathrm{kg})$, and atropine $(0.067 \mathrm{mg} /$ $\mathrm{kg}$ ). Temperature was maintained at $37^{\circ} \mathrm{C}$ with a heating blanket (Harvard Apparatus, Holliston, MA). Because the level of anesthesia can affect ORIS, the heart rate, $\mathrm{pO}_{2}$, and $\mathrm{ETCO}_{2}$ were carefully monitored using a small animal Capnograph (Surgivet, Waukesha, WI) and maintained stable throughout the experiment. To further ensure the consistency of ORIS resulting from the level of anesthetic, the supplementary anesthesia $(0.3 \mathrm{gm} / \mathrm{kg}$ urethane) was delivered as needed, depending on the animal's reflex response to a toe pinch. The electrocardiogram was monitored between leads attached to the right hindlimb and the skin of the scalp. Rats were placed in a stereotactic frame. The skin above the skull was shaved, and the scalp was incised along the midline. The skull over one hemisphere, between lambda and bregma, was thinned using a dental drill. A small hole, $\sim 1 \mathrm{~mm}^{2}$, was made in the thinned skull over somatosensory cortex, and a slit was made in the dura. In a subset of the animals $(n=7)$, to ensure the health of the cortex, ORIS of hindlimb sensory cortex was performed before the induction of interictal spikes. Peripheral stimulation was produced by $2 \mathrm{sec}$ of hindlimb electrical stimulation $(2$ $\mathrm{mA}, 5 \mathrm{~Hz})$. A focal change in reflected light $(0.1 \%)$ was visualized over hindlimb cortex beginning $\sim 500 \mathrm{msec}$ after hindlimb stimulation with event triggered averaging over 100 trials (Mehta et al., 2003), consistent with published work (Sheth et al., 2003).

Electrophysiology. A glass micropipette filled with $1 \% \mathrm{NaCl}$ was positioned at an acute angle $\left(20-40^{\circ}\right)$ to the neocortical surface, $\sim 300 \mu \mathrm{m}$ below the neocortical surface for field potential (F.P.) recording. Signals were digitized at $1000 \mathrm{~Hz}$ by a CED Power 1401 (Cambridge Electronic Design, Cambridge, UK) and recorded by a personal computer. Records were examined for the presence of spreading depression, which was not

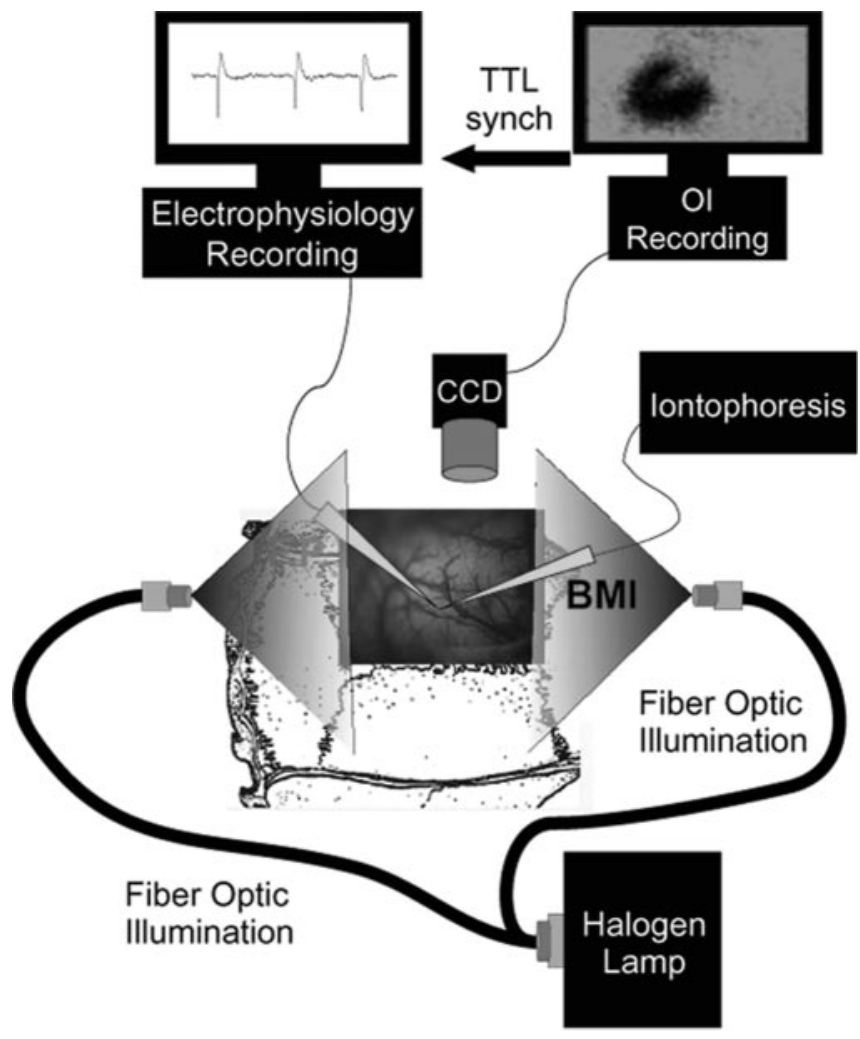

Figure 1. Simultaneous ORIS- electrophysiology set up. Two fiber-optic light guides illuminate the exposed cortex through the thinned skull between lambda and bregma. F.P. and iontophoresis electrodes penetrate the skull and are advanced into the cortex. A time stamp for each frame acquisition with the optical imaging (OI) computer is recorded on the electrophysiology computer for later correlation with the IISS. TTL synch, Transistor-transistor logic synchronization.

found in any of the experiments published in this report. A second glass pipette (tip resistance, $4-6 \mathrm{M} \Omega$ ) was filled with a solution of bicuculline methiodide (BMI) (5 mM in $165 \mathrm{~mm} \mathrm{NaCl}$, pH 3.0; Sigma-Aldrich, St. Louis, MO) and positioned at an acute angle $<1 \mathrm{~mm}$ from the F.P. electrode. Interictal foci were induced by iontophoresis of BMI into layers II-III, using a microiontophoresis dual current generator (World Precision Technology, Sarasota, FL). Currents used were -15 to $-20 \mathrm{nA}$ for retention and +50 to $+500 \mathrm{nA}$ for release depending on the resistance of the micropipette tip. Positive currents were maintained until stereotypical IISs were recorded (after $\sim 5 \mathrm{~min}$ ).

Optical imaging. A CCD camera using a tandem lens $(50 \times 50 \mathrm{~mm})$ arrangement (Ratzlaff and Grinvald, 1991) was focused $400 \mu \mathrm{m}$ below the cortical surface around the area of BMI iontophoresis to deemphasize the pial vasculature in the surface of the cortex. Images were obtained at $10 \mathrm{~Hz}$ during multiple 20 sec blocks over $\sim 400 \mathrm{sec}$ by a 10 -bit video imaging system (Imager 3001; Optical Imaging, Germantown, $\mathrm{NY}$ ). Using this configuration, a $9 \times 7 \mathrm{~mm}$ area of cortex was imaged with $768 \times 480$ pixels and stored with $3 \times 3$ pixel binning. Illumination was performed by filtering a halogen lamp at $546 \pm 10,605 \pm 10,630 \pm$ 10 , and $700 \pm 10 \mathrm{~nm}$, which was homogeneously projected onto the cortex with the aid of two fiber-optic light guides (Fig. 1). The wavelength of light was randomly altered between sets of blocks to avoid bias. At 546 $\mathrm{nm}$, an isosbestic wavelength of hemoglobin (path length corrected) (Sheth et al., 2003), we monitored total hemoglobin (Hbt), which is directly proportional to $\mathrm{CBV}$ and $\mathrm{CBF}$, assuming that the concentration of red blood cells remains constant (Frostig et al., 1990; Mayhew et al., 2000; Nemoto et al., 2004). At higher wavelengths $(600-650 \mathrm{~nm})$, the majority of the signal arises from the oxygenation state of hemoglobin, because $\mathrm{Hbr}$ absorbs light with three times the absorption coefficient as $\mathrm{HBO}_{2}$ (Malonek and Grinvald, 1996; Sato et al., 2002). Hence, a decrease in light reflection indicates an increase in Hbr. We chose to monitor 
A

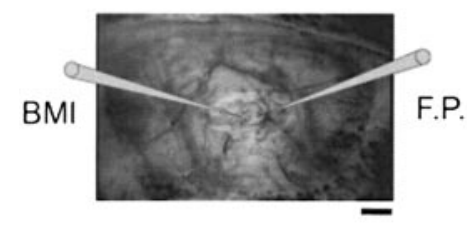

B

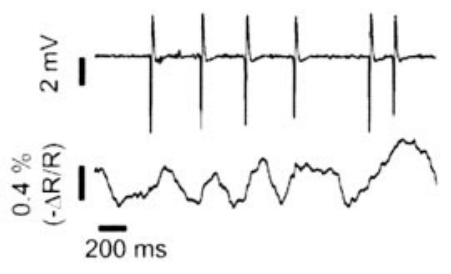

C

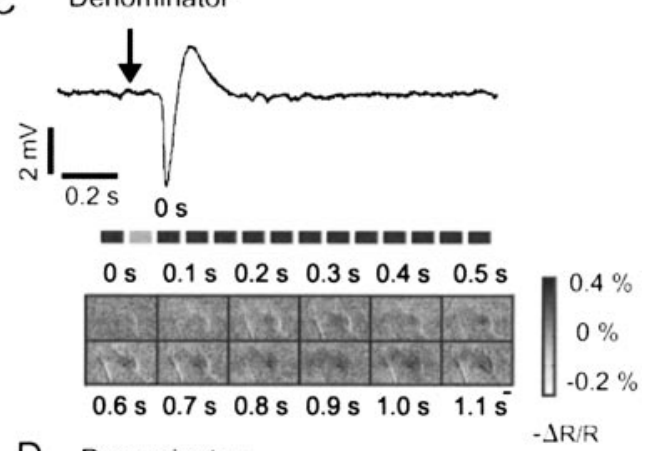

D Denominator

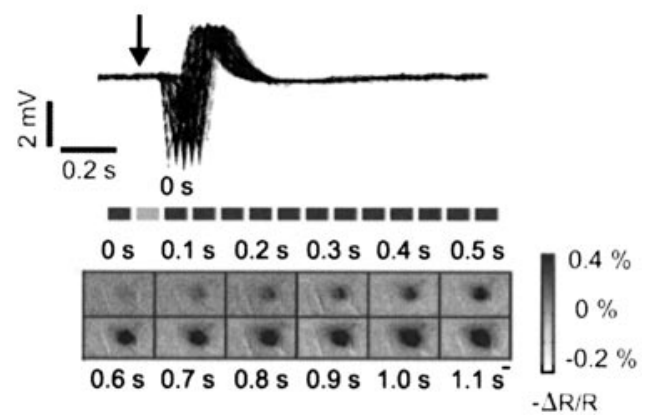

Figure 2. Spike-triggered image division averaged over multiple spikes. IIS epilepsy maps can be generated using spiketriggered image division from either a single IIS or multiple IISs. $A$, Image obtained at $546 \mathrm{~nm}$ shows blood vessel pattern seen through a thin layer of skull. $B$, Stable, well-formed, biphasic IISs were induced at regular intervals with focal iontophoresis of BMI. Simultaneous ORIS and F.P. recordings show that each IIS induces a change in reflection of light. C, ORIS spike-triggered map at $605 \mathrm{~nm}$ was generated by dividing each single frame after the IIS (black bars) by the single frame (gray bar) that preceded the IIS (frame duration, $100 \mathrm{msec}$ ). D, To increase the signal-to-noise ratio, we averaged over multiple spikes ( $n=185$ ). Each IIS crosses a certain threshold within the first $100 \mathrm{msec}$, but the timing of the peak is variable. Frame rate and images are identical to $C$ in the same animal. Scale bar, $1 \mathrm{~mm}$.

blood oxygenation at two wavelengths, 605 and $630 \mathrm{~nm}$, because a variety of wavelengths $600-630 \mathrm{~nm}$ have been used in previous studies, and we wished to investigate the impact of this range on the intrinsic signals associated with epilepsy. In addition, because both of these wavelengths show changes in hemoglobin oxygenation, they act as an internal control to demonstrate the reproducibility of our findings. Early increases in absorption of light are consistent with an increase in $\mathrm{Hbr}$, which we refer to as the initial dip. Later decreases in light absorption, consistent with an increase in $\mathrm{HBO}_{2}$, we heretofore call the BOLD signal, because most fMRI studies done with $1.5 \mathrm{~T}$ magnets are not sensitive enough to pick up the early increase in $\mathrm{Hbr}$. At wavelengths $>650 \mathrm{~nm}$, such as $700 \mathrm{~nm}$ as we used, the signal from LS becomes progressively more significant and ultimately dominates the intrinsic optical signal (IOS) in the nearinfrared region $(>800 \mathrm{~nm})$, particularly in avascular preparations (Malonek and Grinvald, 1996; Sato et al., 2002). Because bandpass filters are $\pm 10 \mathrm{~nm}$, no signal is a pure measure of the underlying physiological event. After insertion of the electrodes, $2 \%$ agar solution (SigmaAldrich), warmed to $38^{\circ} \mathrm{C}$, was poured around the surface of the thinned skull. A glass coverslip was placed on the surface of the liquid agar, with care not to disrupt the intracortical micropipettes.

Data analysis. To quantify the change in light reflectance, the series of images obtained after each IIS event was divided by the image occurring two frames before the IIS event. All quantitative analysis including mean (SD) of pixel values in a region of interest (ROI) was performed using custom image analysis software written in MATLAB. To further increase the signal-to-noise ratio of this spike-triggered image division, averaging over multiple spikes with similar amplitude and interspike intervals was performed (Schwartz and Bonhoeffer, 2001). An automated algorithm was written to include only IISs above a certain threshold and reject those with an ISI $<1$ sec to reduce the influence of previous or subsequent IISs on the optical signal. The percentage of change in light reflectance at each time point was quantified by computing the $-\Delta R / R(\%)$ in an ROI. To

calculate the intrinsic signal associated with the focus, an ROI was selected in the region of IIS onset. The "inverted" optical signals from the putative "inhibitory surround" were selected from multiple separate ROIs at the periphery of the area of decreased light reflectance. The mean (SD) of the pixel values within the ROI was compared before and after the onset of the IIS, and the onset latency was defined as the first frame with a statistically significant change in $-\Delta R / R(\%)$ (paired $t$ test; $p<0.05$ ). After the calculation of the percentage of change in light reflectance in the ROIs, the raw images were spatially filtered using a moving average $5 \times 5$ pixel vector for smoothing. The filtered images were then used to calculate the spatial extent of the IOS and also used for generating figures.

To determine the spatial extent of each IIS event, we used the "normalized threshold analysis" method developed by Chen-Bee and others (Masino et al., 1993; Chen-Bee et al., 1996; Brett-Green et al., 2001). For a given set of images, the dynamic range of pixel intensities (minimum to maximum) was determined. Each pixel was then evaluated to determine whether it was a given percentile of the dynamic range below the median pixel intensity. The median was used instead of the mean, because it is less sensitive to outliers. Note that this procedure can also be used to determine the size of the region of increased light reflectance (i.e., inverted optical signal in the putative inhibitory surround) by evaluating which pixels lay a given percentile of the dynamic range above the median. The determination of the spatial extent can then be performed using any given percentile of the dynamic range ("threshold"). With higher thresholds, the criterion for determining area of the event more stringent, and the absolute size will be smaller. Hence, the calculated area is somewhat arbitrary in an absolute sense. However, comparisons between wavelengths were always made at the same threshold and varying this threshold did not alter the results (see Fig. 8). In this way, the relative size of each event at each wavelength was determined.

\section{Results}

\section{Spike-triggered image averaging of the IIS}

Periodic $(0.15-0.5 \mathrm{~Hz})$ biphasic IISs were induced with BMI iontophoresis and recorded with an adjacent F.P. electrode (Fig. $2 A)$. Mean (SD) amplitude of each IIS $(n=100)$ was $-3.8 \pm 0.4$ $\mathrm{mV}$, and mean (SD) spike width was $85 \pm 18 \mathrm{msec}$. Simultaneous electrocorticography electrodes placed just outside the limits of the region of thinned bone did not record the events, indicating that horizontal propagation was limited. Each IIS induced a change in light reflection that returned near baseline depending on the interspike interval (Fig. $2 B$ ). Spike-triggered image division revealed that the amplitude of the intrinsic signal change was sufficiently large to localize a single IIS, without any image averaging, even at a temporal resolution of $100 \mathrm{msec}$ (Fig. 2C). We did not find a prominent $0.1 \mathrm{~Hz}$ vasomotor artifact in our rat data (Mayhew et al., 1996), which may be dependent on species, anesthesia, and analysis technique (Kalatsky and Stryker, 2003). However, averaging over multiple IISs $(n=185)$ significantly increased the signal-to-noise ratio (Fig. 2D). Compared with the ferret (Schwartz and Bonhoeffer, 2001), where the change in reflection associated with IISs was $\sim 5 \%$, in the rat, the signal was an order of magnitude lower, on the order of $0.3 \%$. This surprisingly 
low value is approximately equivalent to intrinsic signal changes associated with normal sensory processing in the rat (Frostig et al., 1990; Sheth et al., 2003, 2004). This difference may be partially caused by the spike-triggered image division technique, in which the denominator frame has residual signal from the previous IIS.

Because IISs occur spontaneously at irregular intervals but the images are acquired at fixed regular intervals, the peak of each IIS occurs at a slightly different time (Fig. 2D). However, according to the IIS selection algorithm, the downslope of the IIS must cross a certain threshold within the first frame. Despite this variability, in which some IISs do not peak until well into the second $100 \mathrm{msec}$ frame, it is clear that a statistically significant decrease in reflection occurs within $100 \mathrm{msec}$ of the onset of the IIS (Fig. 2D) (paired $t$ test; $p<$ $0.05)$. In the next set of experiments, we investigated the wavelength dependence of the latency and amplitude of the intrinsic signal change to determine the evolution of CBV, Hbr, and LS after the IIS.

\section{Optical mapping of IISs at multiple wavelengths}

Spike-triggered mapping was performed at all four separate wavelengths in individual animals $(n=10)$. An example of such an experiment is presented in Figure 3. The optical signal at $546 \mathrm{~nm}$, an in vivo isosbestic wavelength of hemoglobin (Sheth et al., 2004), demonstrated changes in CBV associated with the IIS (Fig. 3A). A decrease in light reflection, marking an increase in CBV, is noted within $100 \mathrm{msec}$ of the IIS in the region of the iontophoresis, coincident with the location of the population of neurons participating in the IIS. The signal appears in the parenchyma of the cortex and avoids the blood vessels (Fig. $3 A, E$ ). The intensity of the change in reflection, as well as the area, progressively increases and spreads, peaking $\sim 2-2.5 \mathrm{sec}$ after onset (Fig. 3A). A small negative signal is seen surrounding the focal darkening, presumably representing a decrease in CBV. The amplitude and time course of the intrinsic signal changes from an ROI in the focus and four ROIs in the surrounding cortex ( $2 \mathrm{~mm}$ distance) demonstrate that the negative optical signal appears in all quadrants around the focus (Fig. $3 H$ ). The amplitude of this signal is clearly smaller than the positive signal.

At 605 and $630 \mathrm{~nm}$, representing the range of wavelengths used in previous studies to measure hemoglobin oxygenation (Vnek et al., 1999; Devor et al., 2003; Sheth et al., 2003; Vanzetta et al., 2004), our data clearly show (Fig. 3 B, C) a focal increase in
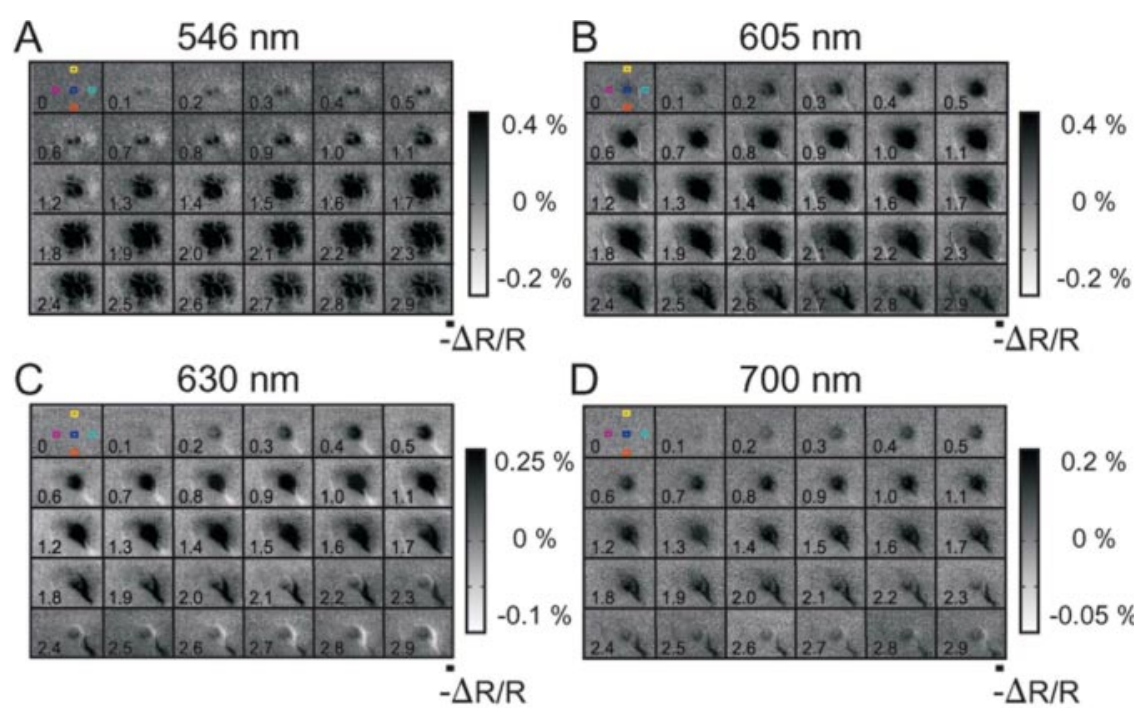

D $\quad 700 \mathrm{~nm}$

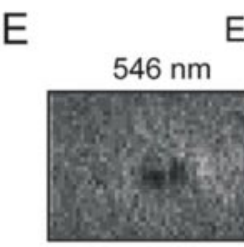

$630 \mathrm{~nm}$

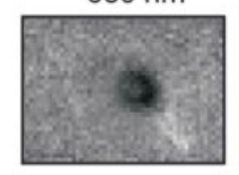

G

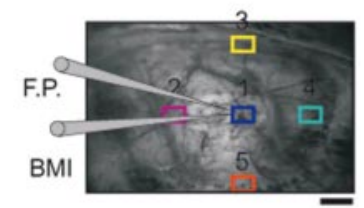

Figure 3. ORIS at multiple wavelengths. $A-D$, Spike-triggered image division at $546 \pm 10 \mathrm{~nm}(A)(n=208$ spikes), $605 \pm 10$ $\mathrm{nm}(B)(n=185$ spikes $), 630 \pm 10 \mathrm{~nm}(C)(n=218$ spikes $)$, and $700 \pm 10 \mathrm{~nm}(D)(n=215$ spikes $)$ demonstrate the wavelength dependence of the optical signal (see Discussion). $E$, Magnified example of spike-triggered image divisions at four wavelengths at $0.3 \mathrm{sec}$ (early) after IISs. F, Magnified example of spike-triggered image divisions at four wavelengths at $2.5 \mathrm{sec}$ (late) after IISs. G, The amplitude and time course of the intrinsic signal change was measured from a single ROI in the focus (dark blue) and multiple, symmetric ROls in the surrounding cortex (red, orange, light blue, yellow). $H$, Inverted optical signals recorded from the surrounding cortex are smaller in amplitude than signals recorded from the focus, regardless of wavelength, and appear in all directions. The amplitude of the signals decreases with increasing wavelength. Scale bars, $1 \mathrm{~mm}$
Hbr after each IIS, which is colocalized with the CBV signal. This "dip" in oxygenation appears within $100 \mathrm{msec}$ of the IIS, spreads horizontally in all directions, and peaks at $\sim 1.5 \mathrm{sec}$ (Fig. $3 H$ ). Like the CBV signal, the decrease in oxygenated hemoglobin was parenchymal and focal at the early stages (Fig. 3E). After $\sim 2 \mathrm{sec}$, 
A

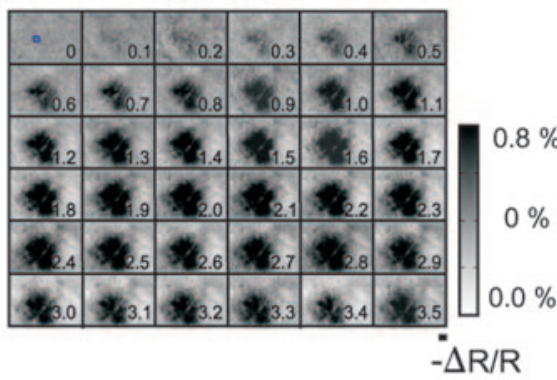

C

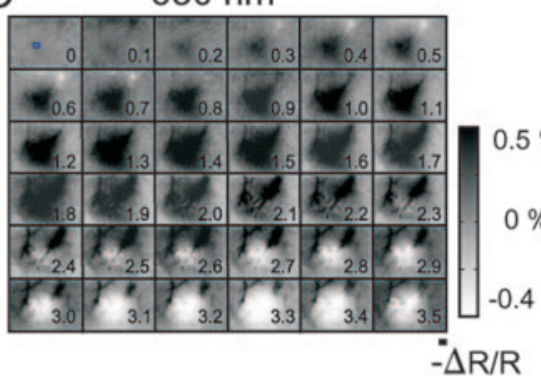

E

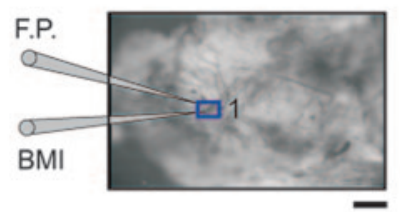

G

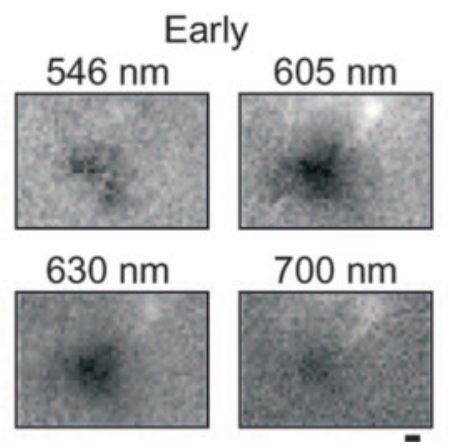

B

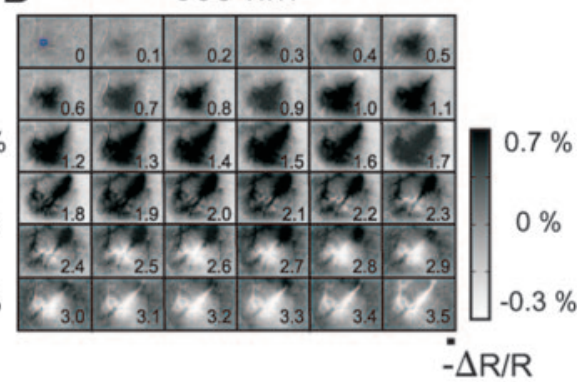

signal, the parenchyma-based negative signal was distributed symmetrically around the focus and was smaller in amplitude than the signal in the focus (Fig. $3 H$ ). A late negative signal was also seen in the large draining veins, starting $\sim 2.5 \mathrm{sec}$ after the IIS (Fig. 3C).

ORIS at $700 \mathrm{~nm}$ was approximately the same as at 605 or $630 \mathrm{~nm}$, except the optical signals in the focus and surrounding cortex were smaller in amplitude and size (Fig. 3D, $H$ ). Quantitative analysis of this data showed that the maximal changes in light reflectance were significantly different at each wavelength [ANOVA; $p<$ 0.001; post hoc Student-Newman-Keuls (SNK) test]. The average maximal values of $-\Delta R / R(\%)$ measured from the ROI in the focus was $0.34 \pm 0.017 \%$ at $546 \mathrm{~nm}$, $0.29 \pm 0.004 \%$ at $605 \mathrm{~nm}, 0.17 \pm 0.003 \%$ at $630 \mathrm{~nm}$, and $0.14 \pm 0.004 \%$ at $700 \mathrm{~nm}$. The average minimal values of $-\Delta R / R$ (\%) measured from surrounding ROIs were $-0.10 \pm 0.01 \%$ at $546 \mathrm{~nm},-0.088 \pm$ $0.02 \%$ at $605 \mathrm{~nm},-0.044 \pm 0.012 \%$ at 630 $\mathrm{nm}$, and $-0.025 \pm 0.007 \%$ at $700 \mathrm{~nm}$ and were not significantly different at each wavelength (ANOVA; $p>0.05$; post hoc SNK test).

\section{Early versus late intrinsic signal}

Because the intrinsic signal is not only sensitive to neuronal action potentials but also to subthreshold activity (Das and Gilbert, 1995), factors that influence the focality of the spatial extent of the signal, such as wavelength and timing, are critical for its localizing value. We present another example of a multiwavelength experiment to highlight the reproducibility of our findings, as well as the variability, and quantify the spatial extent of the positive and negative signal over time. In this ani$\mathrm{mal}$, as in the previous example, a decrease in reflection of light was identified at all wavelengths within $100 \mathrm{msec}$ of the IIS that initiated focally at the site of the iontophoresis and then spread horizontally (Fig. 4). The CBV signal recorded at 546 $\mathrm{nm}$ remained parenchymal, with little involvement of the vasculature (Fig. $4 A, G, H)$. At wavelengths sensitive to the oxygenation of hemoglobin (605 and $630 \mathrm{~nm})$, the signal began in the paren$\mathrm{nm}(A)(n=134$ spikes), $605 \pm 10 \mathrm{~nm}(B)(n=125$ spikes), $630 \pm 10 \mathrm{~nm}(C)(n=75$ spikes $)$, and $700 \pm 10 \mathrm{~nm}(D)(n=73$ spikes) highlights the reproducibility and variability of our results. Note the late inverted $10 S$ at 605 and $630 \mathrm{~nm}$ begins focally and then propagates in the draining veins. $E$, The image at $546 \mathrm{~nm}$ shows a blood vessel pattern of an imaged region covered with thin skull. The blue box is an ROI in focus measured in F. Scale bars, $1 \mathrm{~mm}$. Time course of superpixel in the focus measured at each wavelength. G, Magnified example of spike-triggered image divisions at four wavelengths at $0.3 \mathrm{sec}$ (early) after IISs. H, Magnified example of spike-triggered image divisions at four wavelengths at $3.0 \mathrm{sec}$ (late) after IISs. Scale bars, SEM.

this signal moved into the draining veins (Fig. $3 B, C, F$ ). At oxygen-sensitive wavelengths, an inverted signal, or increase in light reflectance, marks an increase in oxygenated hemoglobin, which is described in the fMRI literature as an increase in BOLD signal. We noted two examples of this phenomenon: (1) parenchyma-based increases in light reflection and (2) blood vessel (venous)-based increases in light reflection. An early increase in oxygenation was seen both focally in the draining veins and diffusely in the parenchyma surrounding the focus. Like the CBV chyma and terminated in the draining veins after $1 \mathrm{sec}$ (Fig. $4 B, C, G, H)$. Once again, a highly localized dip in oxygenation is clearly recorded from the focus. Inverted optical signals were also recorded from the surrounding parenchyma at all wavelengths, and at 605,630 , and $700 \mathrm{~nm}$, an early $(100-700 \mathrm{msec})$ transient signal was seen in the draining veins. In this example, unlike the first example, a late $(>2 \mathrm{sec})$ inverted signal appeared in the region of the focus that then transitioned into the draining veins, consistent with a prominent increase in BOLD signal in the focus 


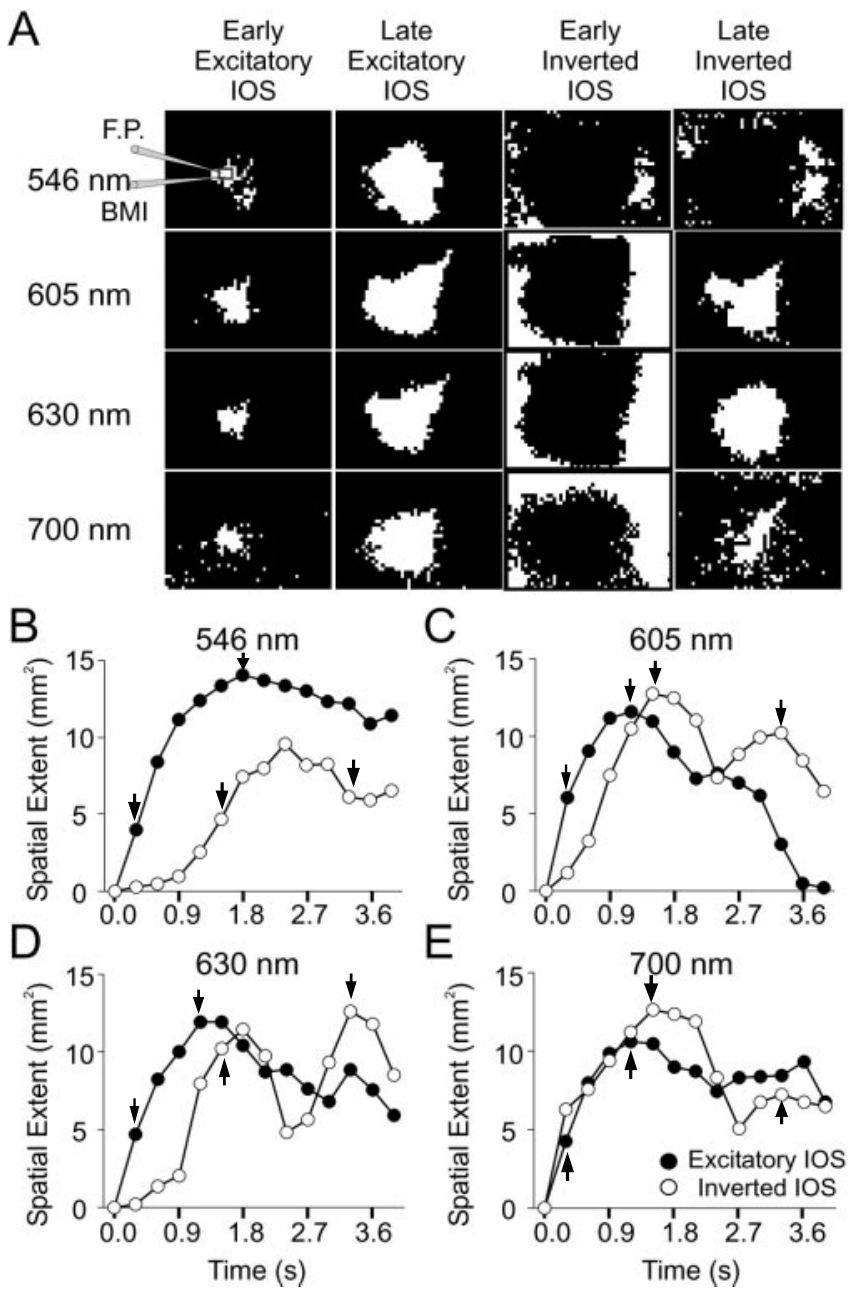

Figure 5. Spatial extent of IOS as a function of wavelength. Spatial extents were calculated by thresholding pixels as described in Materials and Methods. $A$, Spatial extents of both excitatory IOS in focus and inverted IOS in surround have wavelength dependence. The white area indicates pixels for which values are below (decrease in reflection of light) or above (increase in reflection of light) the threshold for excitatory and inverted signals, respectively. Early excitatory IOS was chosen $0.3 \mathrm{sec}$ after the IIS, whereas late excitatory IOS was chosen at the peak of spatial extent at each wavelength. The inverted IOS was thresholded at the maximum of the early and late peaks; in this example, these peaks occurred at 1.5 and 3 sec after the IIS, respectively. The timing of images is denoted by arrows in $B-E$. Note the focality of the IOS in the focus recorded early and the inversion of the inverted optical signal in surround comparing early to late images. Scale bars, $1 \mathrm{~mm}$. The time course of spatial extent for both excitatory (closed circle) and inverted (open circle) IOS is demonstrated at $546 \mathrm{~nm}(B), 605 \mathrm{~nm}(C), 630 \mathrm{~nm}(D)$, and $700 \mathrm{~nm}(E)$.

(Fig. $4 H$ ). Measurements of $-\Delta R / R(\%)$ from an ROI in the focus demonstrate that the signal amplitude decreases with increasing wavelength, and the monophasic CBV signal peaks later than the biphasic oxygenation signal (Fig. $4 F$ ). Although the initial dip in oxygenation is highly localized at its early stages, in this example, we see a later increase in oxygenation that is also highly localized in its early stages, before transitioning to the less welllocalized draining veins.

At each wavelength, the spatial extent of the IOS, both positive and negative, was calculated using the normalized threshold analysis technique with a threshold set $10 \%$ above and below the median, respectively (see Materials and Methods for additional details). As is clearly seen in Figure $5 A$, the early changes in the optical signal, which occur at $300 \mathrm{msec}$ after the IIS, are much more focal than the late changes at the peak of the optical signal
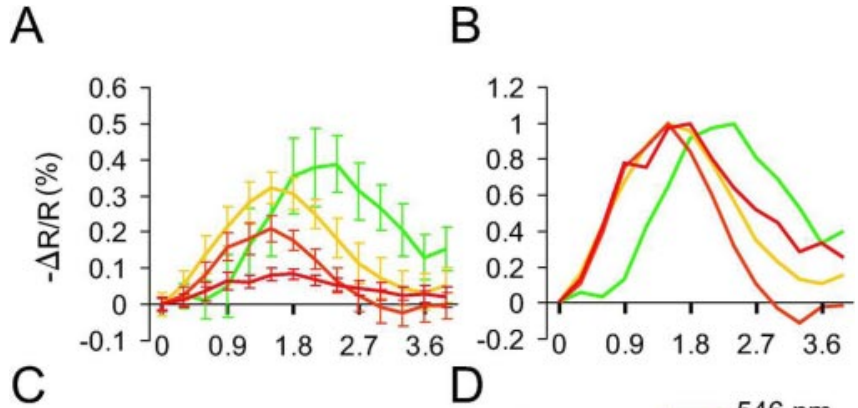

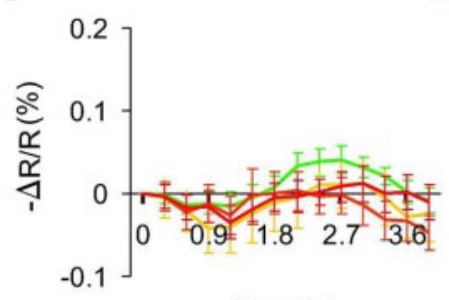

Time (s)

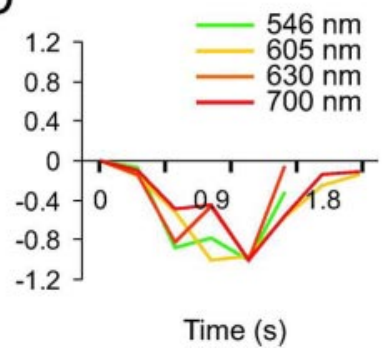

Figure 6. Average results of changes in reflectance as a function of time. $A$, Percentage of change in reflection of light in the focus as a function of time and wavelength after each IIS. The amplitude of the 10 is clearly greatest when recorded at $546 \mathrm{~nm}$, followed by 605,630 , and 700 $\mathrm{nm} . B$, Data are normalized to the maximum change in $-\Delta R / R(\%)$ to show that the rise in the signal is fastest at higher wavelengths. C, Percentage of change in reflection of light from the surround as a function of time and wavelength after each IIS. An inverted optical signal is seen in all wavelengths, and the amplitude of this inverted IOS is equivalent, regardless of wavelength. $D$, Data are normalized to the maximum negative change in $-\Delta R / R(\%)$ to show that, temporally, there is no wavelength dependence in this signal.

( $>1 \mathrm{sec})$. In this particular example, at $300 \mathrm{msec}$ after the IIS, the spatial extent of the positive optical signal in the focus was similar at all wavelengths (Fig. 5). At the peak of the optical signal, however, the spatial extent at $546 \mathrm{~nm}$ was slightly larger than the other wavelengths, and the signal clearly spreads into the draining veins at oxygen-sensitive wavelengths (Fig. 5). The inverted optical signal in the surround shows an equally dramatic temporal dependence and actually reverses at higher wavelengths when the early and late phases are compared (Fig. 5). At wavelengths sensitive to $\mathrm{CBV}$, the inverted signal gradually spreads over time in the surrounding brain tissue. At oxygen-sensitive wavelengths, however, the inverted signal has two separate peaks, an earlier one in the surrounding cortex at $1.5 \mathrm{sec}$ and a later one in the focus at 3 sec (Fig. 5). This animal provided a dramatic example of a late increase in oxygenated hemoglobin within the focus that might be demonstrable using conventional increase in BOLD fMRI. However, this was only observed in 2 of 10 animals. There was a nonsignificant trend toward a higher frequency of IISs in the animals without a prominent increase in BOLD signal in the focus but only in the draining veins $(0.33 \pm 0.08 \mathrm{~Hz})$ compared with animals that did have an increase in BOLD signal within the focus $(0.19 \pm 0.06 \mathrm{~Hz} ; p=0.08)$.

\section{Average amplitude and latency of IOS}

The average amplitude of IOS $[-\Delta R / R(\%)](n=10$ animals $)$ over the interictal focus at its peak was $0.38 \pm 0.11(546 \mathrm{~nm})$, $0.32 \pm 0.05(605 \mathrm{~nm}), 0.20 \pm 0.04(630 \mathrm{~nm})$, and $0.09 \pm 0.02$ $(700 \mathrm{~nm})$. All differences are statistically significant (ANOVA; post hoc SNK test; $p<0.05$ ) (Fig. $6 A$ ). The CBV signal $(546 \mathrm{~nm}$ ) was not only larger in amplitude but also peaked later than the signal recorded at higher wavelengths, reaching its maximum at $\sim 2.5 \mathrm{sec}$ versus $\sim 1.5 \mathrm{sec}$ for the oxygen-dependent and LS signal (Fig. 6A). In contrast, the "early" signal was actually larger in 


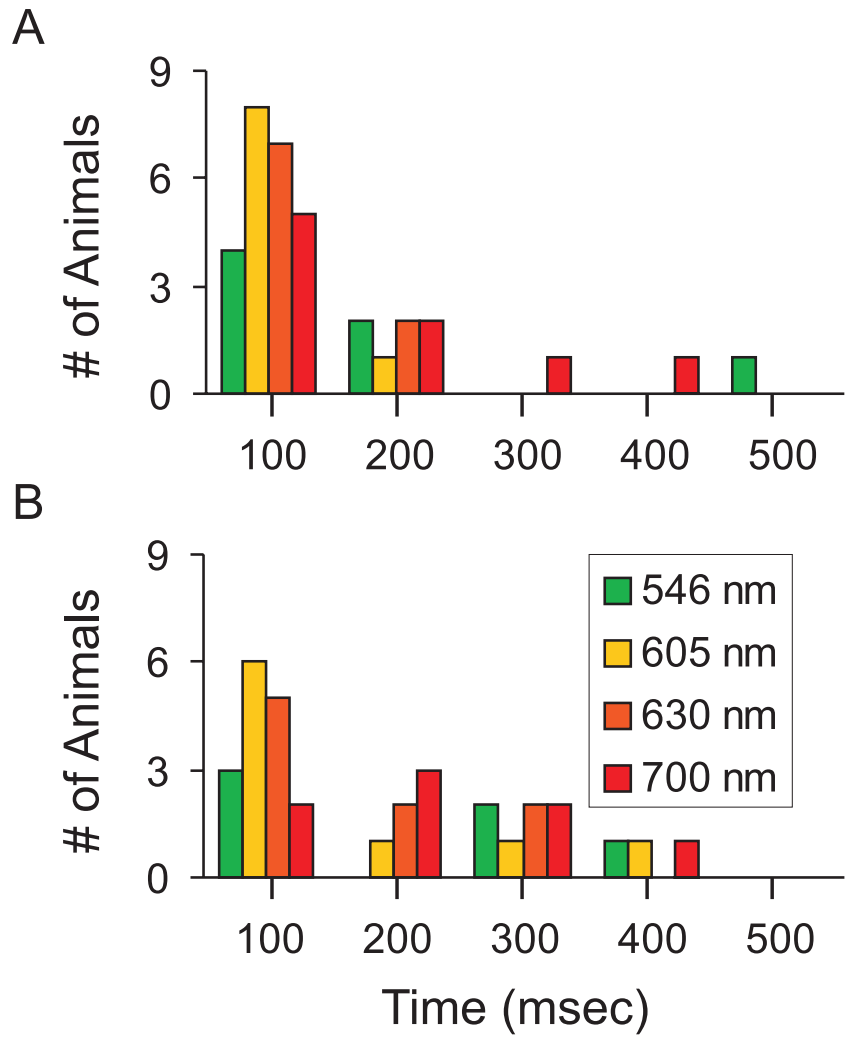

Figure 7. Earliest significant change in light reflection. $A$, The earliest statistically significant change (paired $t$ test; $p<0.05$ ) in reflectance of light was seen at $100 \mathrm{msec}$ at all wavelengths, although more commonly at wavelengths sensitive to oxygenation of hemoglobin. $B$, The earliest statistically significant (paired ttest; $p<0.05$ ) inverted change in reflectance of light in the surround was seen at $100 \mathrm{msec}$, regardless of the wavelength. Again, the oxygenation signal is more commonly seen at $100 \mathrm{msec}$ than the CBV signal. In several cases, the inverted signal in the surrounding cortex was delayed compared with the positive signal in the focus for all wavelengths tested.

amplitude at oxygen-sensitive wavelengths (Fig. 6A). When normalized to the maximum amplitude of IOS value, the onset of the signal is clearly fastest at higher wavelengths (Fig. $6 \mathrm{~B}$ ). However, regardless of wavelength, a statistically significant change in the optical signal was found within $100 \mathrm{msec}$ of the IIS in $40-80 \%$ of the animals (paired $t$ test; $p<0.05$ ) (Fig. 7A). On average, the onset occurred at $186 \mathrm{msec}(546 \mathrm{~nm}), 111 \mathrm{msec}(605 \mathrm{~nm}), 122$ $\mathrm{msec}(630 \mathrm{~nm})$, and $178 \mathrm{msec}(700 \mathrm{~nm})$. Thus, the fastest signal change was most consistently found at higher wavelengths sensitive to both hemoglobin oxygenation and LS.

Unlike the amplitude and latency of excitatory IOS, the amplitude and latency of the inverted optical signal in the surround did not show any clear wavelength dependence (Fig. $6 \mathrm{C}$ ), which can also be seen when normalized to the maximal amplitude of the inverted signal (Fig. 6D). An ANOVA test showed that the peak amplitude of the inverted IOS was not significantly different at all four wavelengths ( post hoc SNK test; $p>0.05$ ). The inverted signal peaked $1 \mathrm{sec}$ after the IIS for all wavelengths, which was, on average, earlier than the peak of the positive signal, although occasional exceptions were found (Fig. 3, $546 \mathrm{~nm}$ ). Like the positive signal, the inverted signal was also recorded as early as 100 msec in $30-60 \%$ of the animals (paired $t$ test, $p<0.05$ ) (Fig. $7 B$ ). On average, however, the onset occurred at $225 \mathrm{msec}(546 \mathrm{~nm})$, $180 \mathrm{msec}(605 \mathrm{~nm}), 175 \mathrm{msec}(630 \mathrm{~nm})$, and $247 \mathrm{msec}(700 \mathrm{~nm})$, which was consistently slightly delayed compared with the positive signal from the focus.
A
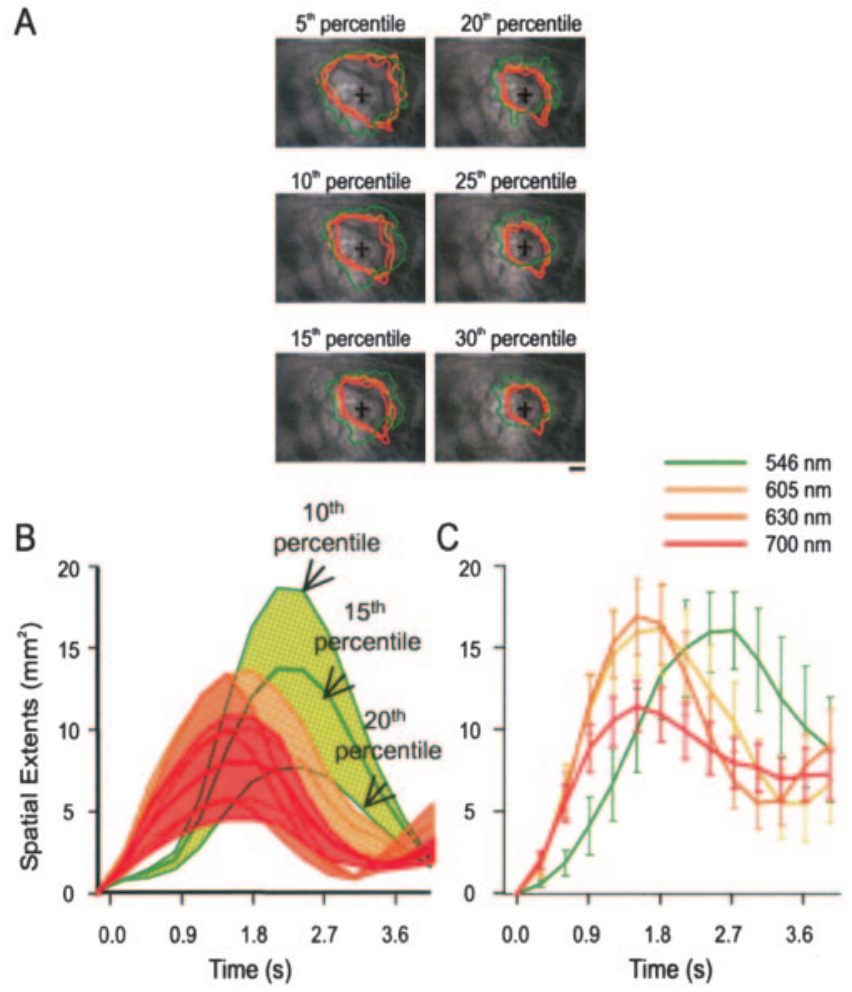

Figure 8. Spatial extent of excitatory $10 S$ as a function of time and wavelength. $A$, Each panel depicts a contour plot of the spatial extent of an average spike-triggered map at different wavelengths and thresholds. Although the absolute area and shape change with the threshold, the relative size remains stable. Scale bars, $1 \mathrm{~mm}$. B, Time course of spatial extent at 5, 10, and $15 \%$ threshold in one animal. The temporal dependence on the relative sizes is apparent regardless of threshold. $C$, The average extent of spread (area) of the change in reflectance in the focus in all animals $(n=10)$ is both temporally and wavelength dependent. The slope of the graph in the first 1 sec demonstrates the rate of propagation of the signal.

\section{Average spatial extent and propagation velocity of IOS}

The calculation of an area using a thresholding technique is dependent on the chosen threshold. To ensure that our results were not biased by the arbitrariness of this choice, we investigated the relative area of the IOS at multiple thresholds and wavelengths ( $n=10$ animals). As we varied the threshold, the absolute size and shape of the IOS changed, but the relative area for each wavelength was stable (Fig. $8 \mathrm{~A}$ ). Depending on the latency of the measurement, the relative size of the optical signal varied dramatically between wavelengths (Fig. 8C). Within $1 \mathrm{sec}$ of the IIS, the CBV signal $(546 \mathrm{~nm})$ was significantly smaller than the other wavelengths (Fig. $8 C$, Table 1). From 1-2 sec, the area was largest at oxygen-dependent wavelengths ( 605 and $630 \mathrm{~nm}$ ), followed by the light scatter wavelength $(700 \mathrm{~nm})$ and then the CBV signal (Fig. 8C, Table 1). After $2.5 \mathrm{sec}$, the CBV signal was the largest, whereas the signal at other wavelengths began to return to baseline (Fig. $8 C$, Table 1). On average, the maximal area of the OIS at $546 \mathrm{~nm}$ was no larger than at 605 or $630 \mathrm{~nm}$. In six animals (60\%), however, the area of spread was significantly larger at $546 \mathrm{~nm}$ (ANOVA; post hoc SNK test; $p<0.05$ ).

To calculate the propagation velocity, we assumed the initial IOS spread was circular and performed a linear fit to calculate the slope of the radius of its spatial extent. Over the first $1 \mathrm{sec}$, the average slopes were $0.24 \pm 0.12 \mathrm{~mm} / \mathrm{sec}\left(R^{2}=0.94 \pm 0.05\right)$ at $546 \mathrm{~nm}, 0.46 \pm 0.15 \mathrm{~mm} / \mathrm{sec}\left(R^{2}=0.91 \pm 0.08\right)$ at $605 \mathrm{~nm}$, $0.44 \pm 0.10 \mathrm{~mm} / \mathrm{sec}\left(R^{2}=0.95 \pm 0.04\right)$ at $630 \mathrm{~nm}$, and $0.43 \pm$ $0.15 \mathrm{~mm} / \mathrm{sec}\left(R^{2}=0.94 \pm 0.06\right)$ at $700 \mathrm{~nm}$. The initial velocity at 
Table 1. Statistical significance in the area of IOS depending on wavelength and latency

\begin{tabular}{|c|c|c|c|c|c|c|c|c|c|c|c|c|c|}
\hline \multirow[b]{2}{*}{ Wavelength } & \multicolumn{13}{|l|}{ Time } \\
\hline & $0.3 \mathrm{sec}$ & $0.6 \mathrm{sec}$ & $0.9 \mathrm{sec}$ & $1.2 \mathrm{sec}$ & $1.5 \mathrm{sec}$ & $1.8 \mathrm{sec}$ & $2.1 \mathrm{sec}$ & $2.4 \mathrm{sec}$ & $2.7 \mathrm{sec}$ & $3.0 \mathrm{sec}$ & $3.3 \mathrm{sec}$ & $3.6 \mathrm{sec}$ & $3.9 \mathrm{sec}$ \\
\hline 546 versus $605 \mathrm{~nm}$ & $X$ & $X$ & $X$ & $X$ & $X$ & & & & $X$ & $X$ & $X$ & & \\
\hline 546 versus $630 \mathrm{~nm}$ & & $X$ & $X$ & $X$ & $X$ & & & $X$ & $X$ & $X$ & $X$ & & \\
\hline 546 versus $700 \mathrm{~nm}$ & & $X$ & $X$ & $X$ & & & & $X$ & $X$ & $X$ & $X$ & & \\
\hline 605 versus $630 \mathrm{~nm}$ & & & & & & & & & & & & & \\
\hline 605 versus $700 \mathrm{~nm}$ & & & & $X$ & $X$ & $X$ & & & & & & & \\
\hline 630 versus $700 \mathrm{~nm}$ & & & & $X$ & $X$ & $X$ & & & & & & & \\
\hline
\end{tabular}

Summary of ANOVA (post hoc SNK) test of spatial extent of excitatory IOS. X denotes statistical significance $(p<0.05)$.

$546 \mathrm{~nm}$ was significantly slower than the one at 605, 630, and 700 $\mathrm{nm}$ (ANOVA; post hoc SNK test; $p<0.05$ ), but there was no significant difference in the rate of spread between 605, 630, and $700 \mathrm{~nm}$ (ANOVA; post hoc SNK test; $p>0.05$ ).

\section{Discussion}

This report marks the first optical recording of epileptiform activity at wavelengths sensitive to $\mathrm{Hbt}(\mathrm{CBV})$ and $\mathrm{Hbr}$. Haglund et al. (1992) recorded at $690 \mathrm{~nm}$ in the human, Schwartz (2003) and Schwartz and Bonhoeffer (2001) recorded at $707 \mathrm{~nm}$ in the ferret, and Chen et al. (2000) recorded at $850 \mathrm{~nm}$ in the rat. Although the intrinsic signal is thought to be a "slow" signal, insensitive to rapid electrophysiological events, we demonstrated that changes in the IOS occur within $100 \mathrm{msec}$ of an epileptiform event, regardless of wavelength. Even at $546 \mathrm{~nm}$, which is most sensitive to changes in CBV and is believed to be the slowest signal, we found that a majority of experiments show significant changes in light reflectance within $100 \mathrm{msec}$. Our findings are significant not only in elucidating the latency and spatial specificity of the coupling and uncoupling of CBV and Hbr after the IIS but also in having clinical implications for flow-metabolism-based epilepsymapping techniques such as PET, SPECT, and fMRI.

\section{The epileptic initial dip}

At wavelengths sensitive to $\mathrm{Hbr}$, we found a clear decrease in oxygenation in the focus, which began within $100 \mathrm{msec}$ after the IIS that persisted for almost $3 \mathrm{sec}$. The most likely explanation for this event is that the increased metabolic demand of the neuronal population participating in the IIS outweighs the ability of the brain to increase perfusion to the area. The existence of this initial dip during normal somatosensory processing has been challenged based on its absence under certain experimental conditions (Silva et al., 2000; Lindauer et al., 2001). Because oxygendependent signals are extremely sensitive to $\mathrm{pO}_{2}, \mathrm{pCO}_{2}$, and anesthetic depth, some investigators have questioned whether the initial dip is an artifact of the experimental paradigm. In addition, the dip may depend on the method of stimulation (physiologic vs nonphysiologic) analysis technique and variability in path length of light measured at different wavelengths (Harel et al., 2002; Lindauer et al., 2003). However, investigations into the initial dip have all been done after peripheral activation of visual and somatosensory cortex using ORIS or fMRI, and no data exists on the initial dip after epileptiform events. Indeed, previous fMRI studies of epilepsy have generally found an increase in BOLD signal, implying that CBF oversupplies the epileptic focus with $\mathrm{HBO}_{2}$ (Krakow et al., 2001; Lemieux et al., 2001a,b; Benar et al., 2002; Nersesyan et al., 2004; Tenney et al., 2004).

Epilepsy is a pathological condition, and the metabolic demands associated with the IIS are "supranormal." We found that the IIS induces a profound dip in hemoglobin oxygenation (in- crease in Hbr) and, in contrast to normal physiological events, the amplitude of the initial dip was larger than the later increase in oxygenation. In fact, this late increase or BOLD effect was not often seen in the focus at all. One physiological explanation for the lack of a late hyperoxygenation is that the frequency of the interictal events was relatively high, so the metabolic rate in the focus remained supranormal, and there was insufficient time for the brain to adequately perfuse the area with oxygenated blood. This implies that the neurons in an epileptic focus may be chronically hypoxic, depending on the rate of the interictal events. In human epilepsy, however, interictal events are less frequent and less focal, so our findings may be specific to this acute disinhibition rat model. In support of this explanation, previous autoradiography studies in status epilepticus demonstrate that metabolism remains supranormal for several hours, revealing that increases in perfusion ultimately fail to match increases in metabolic demand leading to tissue injury and neuronal damage (Ingvar and Siesjo, 1983; Ingvar, 1986; Tanaka et al., 1990; Kreisman et al., 1991; Pereira de Vasconcelos et al., 2002).

\section{The inverted optical signal}

The significance of the inverted optical signal surrounding the epileptic focus has been debated. It was first described by Haglund et al. (1992) at $690 \mathrm{~nm}$ following electrically triggered afterdischarges in human cortex and attributed to "shunting of blood" from the adjacent brain to the epileptic focus, although they were recording at wavelengths sensitive to light scatter. At higher wavelengths (707 nm), Schwartz and Bonhoeffer (2001) found a similar inverted signal around an acute disinhibited focus in the ferret, which correlated spatially with a decrease in the firing of extracellularly recorded neurons. They postulated that the inverted signal represented the inhibitory surround, a phenomenon first described by Prince and Wilder (1967), characterized by IPSPs in neurons surrounding the penicillin focus in cats. Support for this theory emerged from IOS recording from mouse visual cortex at $707 \mathrm{~nm}$ where, again, an inverted optical signal correlated spatially with a decrease in firing of extracellularly recorded neurons (Schuett et al., 2002).

This is the first report of an inverted optical signal surrounding an epileptic focus in the rat. Using high temporal resolution ORIS, we investigated the spatiotemporal dynamics of the inverted optical signal and describe an early and a late component at wavelengths $>600 \mathrm{~nm}$. The early inverted signal in the surrounding brain appears within $100 \mathrm{msec}$ of the interictal event both symmetrically within the parenchyma of the surrounding brain as well as in the draining veins and lasts $\sim 2 \mathrm{sec}$. The late component, however, occurs $>2 \mathrm{sec}$ within the focus in a subset of animals and then reappears in the draining veins in all cases. Whereas the later inverted optical signal is consistent with the increase in BOLD signal, a perfusion-related phenomenon, the 
early signal is more likely to correspond with a neuronal inhibitory surround.

We also report a novel finding that an inverted optical signal is also apparent in the surrounding brain at $546 \mathrm{~nm}$, implying a ring of decreased CBV around the focus. Such a decrease likely represents shunting of blood to the adjacent active focus. How can we explain an early decrease in Hbr and a decrease in CBV in the surrounding brain immediately after the IIS? One explanation is that the metabolic rate of the neurons decreases out of proportion with the movement of blood into the focus. In other words, Hbt decreases but Hbr decreases even more, which could be explained by a decrease in neuronal activity, or "surround inhibition." Hence, even in the surround, where neurons are inhibited, there is an uncoupling of neuronal $\mathrm{CMRO}_{2}$ and hemoglobin oxygenation, because Hbr decreases out of proportion with demand.

\section{ORIS of epileptiform events: the influence of wavelength and timing on focality}

The spatial correlation between intrinsic signal changes and neuronal activity has been well investigated after sensory processing, particularly in cat visual and rodent barrel cortex (Frostig et al., 1990; Bonhoeffer and Grinvald, 1991; Hübener et al., 1997; Erinjeri and Woolsey, 2002; Sheth et al., 2004). Although the initial deoxygenation signal is the most spatially restricted, recent evidence suggests that the CBV signal is also spatially restricted if measured early in the evolution of the signal $(<2-3 \mathrm{sec})$ and has the advantage of a higher signal-to-noise ratio (Sheth et al., 2003, 2004; Vanzetta et al., 2004). Our data expand this finding to the situation of epileptiform events, because the early maps at 546 $\mathrm{nm}$ were more focal than the maps obtained at higher wavelengths. In fact, the horizontal propagation of the signal at 546 $\mathrm{nm}$ was also the slowest, so the window of opportunity for obtaining highly localized signals was also the longest. The existence of a focal, early increase in CBV was hypothesized in previous studies based on the absence of a complementary decrease in $\mathrm{HBO}_{2}$ during the initial dip (Malonek and Grinvald, 1996; Buxton, 2001; Jones et al., 2001). We directly demonstrate that a rapid focal increase in $\mathrm{CBV}$, more rapid than previously described in studies of somatosensory processing, follows epileptiform events. Thus, CBV-weighted fMRI, which has already been used for mapping sensory architecture (Mandeville et al., 1999a,b), may be useful in mapping epileptiform events, although the amplitude of this signal is smaller than the initial dip in the first 1-2 sec.

Our data show that during the initial stages of its evolution, when the IOS is most focal, the amplitude is larger at wavelengths sensitive to the oxygenation state of hemoglobin. Thus, although focal maps can be obtained at all wavelengths, the most reliable epilepsy maps were obtained from the initial dip. In contrast, when the temporal evolution of the signal is ignored, the signal recorded at $700 \mathrm{~nm}$, which presumably arises mostly from LS, is clearly the most focal. The origins of this signal are multifactorial and have been attributed to the movement of sodium and potassium ions in and out of both neurons and glia associated fluid shifts and increases and decreases in the volume of the extracellular space as well as the morphology of the vasculature (Hill and Keynes, 1949; MacVicar and Hochman, 1991; Bonhoeffer and Grinvald, 1996; Sato et al., 2002). Although LS is present at all wavelengths, the contributions of the signal arising from hemoglobin decrease with increasing wavelength. In our experiments, the LS data were similar to the data recorded at wavelengths sensitive to $\mathrm{Hbr}$, but the optical signal was smaller in amplitude and area. We conclude that LS may not be a significant contributor to the intrinsic signal in vivo, at least at $700 \mathrm{~nm}$, which is consistent with previous studies (Sato et al., 2002). Alternatively, the LS signal may have a similar time course as the Hbr signal after the IIS, which would be surprising because the LS signal has been shown to have a unique temporal profile after normal physiologic sensory stimulation (Malonek and Grinvald, 1996). Ultimately, higher wavelengths may be required to eliminate any hemoglobin-related signal in vivo, because $\mathrm{Hbr}$ and $\mathrm{HBO}_{2}$ have different absorption spectra up to $\sim 800 \mathrm{~nm}$.

In conclusion, we found that the intrinsic signal changes associated with interictal epileptiform events occur more rapidly than previously thought, regardless of wavelength. This increases its utility in mapping the onset of fast events such as interictal spikes. Although the CBV signal is focal in the first $2 \mathrm{sec}$ after the IIS, the amplitude of the initial deoxygenation-related signal is larger, making this signal potentially more useful in mapping the onset of the event in clinical situations such as spike-triggered fMRI and intraoperative ORIS. This extended dip in oxygenation in which metabolic demand and hemoglobin oxygenation are uncoupled is more profound than after normal sensory activation, which is likely a result of the high metabolic rate of the epileptic neurons. We find an inverted optical signal surrounding the focus that has a distinct temporal signature, an early wavelength-independent phase that may be related to neuronal inhibition and a later wavelength-dependent phase, where the signal transitions to the draining veins at wavelengths sensitive to hemoglobin oxygenation like the BOLD signal recorded with fMRI.

\section{References}

Benar CG, Gross DW, Wang Y, Petre V, Pike B, Dubeau F, Gotman J (2002) The BOLD response to interictal epileptiform discharges. NeuroImage 17:1182-1192.

Bonhoeffer T, Grinvald A (1991) Iso-orientation domains in cat visual cortex are arranged in pinwheel-like patterns. Nature 353:429-431.

Bonhoeffer T, Grinvald A (1996) Optical imaging based on intrinsic signals: the methodology. In: Brain mapping: the methods (Toga AW, Mazziota JC, eds), pp 55-99. San Diego: Academic.

Brett-Green BA, Chen-Bee CH, Frostig RD (2001) Comparing the functional representations of central and border whiskers in rat primary somatosensory cortex. J Neurosci 21:9944-9954.

Buxton RB (2001) The elusive initial dip. NeuroImage 13:953-958.

Chen JWY, O'Farrell AM, Toga AW (2000) Optical intrinsic signal imaging in a rodent seizure model. Neurology 55:312-315.

Chen-Bee CH, Kwon MC, Masino SA, Frostig RD (1996) A real extent quantification of functional representations using intrinsic signal optical imaging. J Neurosci Methods 68:27-37.

Das A, Gilbert CD (1995) Long-range horizontal connections and their role in cortical reorganization revealed by optical recording of cat primary visual cortex. Nature 375:780-784.

de Curtis M, Avanzini G (2001) Interictal spikes in focal epileptogenesis. Prog Neurobiol 63:541-567.

Devor A, Dunn AK, Andermann ML, Ulbert I, Boas DA, Dale AM (2003) Coupling of total hemoglobin concentration, oxygenation, and neural activity in rat somatosensory cortex. Neuron 39:353-359.

Duncan JS (1997) Imaging and epilepsy. Brain 120:339-377.

Engel JJ (1990) Functional explorations of the human epileptic brain and their therapeutic implications. EEG Clin Neurophysiol 76:296-316.

Erinjeri JP, Woolsey TA (2002) Spatial integration of vascular changes with neural activity in mouse cortex. J Cereb Blood Flow Metab 22:353-360.

Fox PT, Raichle ME (1986) Focal physiological uncoupling of cerebral blood flow and oxidative metabolism during somatosensory stimulation in human subjects. Proc Natl Acad Sci USA 83:1140-1144.

Frannson P, Kruger G, Merboldt KD, Frahm J (1998) Temporal characteristics of oxygenation-sensitive MRI responses to visual activation in humans. Magn Res Med 39:912-919.

Frostig RD, Lieke EE, Ts'o DY, Grinvald A (1990) Cortical functional architecture and local coupling between neuronal activity and the microcircu- 
lation revealed by in vivo high-resolution optical imaging of intrinsic signals. Proc Natl Acad Sci USA 87:6082-6086.

Granquist-Fraser D, Polimeni J, Schwartz EL (2003) Physical limits to spatial resolution of optical recording: photon scatter and optical defocus. Soc Neurosci Abstr 29:125.3.

Haglund MM, Ojemann GA, Hochman DW (1992) Optical imaging of epileptiform and functional activity in human cerebral cortex. Nature 358:668-671.

Harel N, Lee SP, Nagaoka T, Kim DS, Kim SG (2002) Origin of negative blood oxygenation level-dependent fMRI signals. J Cereb Blood Flow Metab 22:908-917.

Hill DK, Keynes RD (1949) Opacity changes in stimulated nerve. J Physiol (Lond) 108:278-281.

Hübener M, Shoham D, Grinvald A, Bonhoeffer T (1997) Spatial relationships among three columnar systems in cat area 17. J Neurosci 17:9270-9284.

Ingvar M (1986) Cerebral blood flow and metabolic rate during seizures: relationship to epileptic brain damage. Ann NY Acad Sci 462:207-223.

Ingvar M, Siesjo BK (1983) Local blood flow and glucose consumption in the rat brain during sustained bicuculline-induced seizures. Acta Neurol Scand 68:129-144.

Jones M, Berwick J, Johnston D, Mayhew JEW (2001) Concurrent optical imaging spectroscopy and laser-doppler flowmetry: the relationship between blood flow, oxygenation, and volume in rodent barell cortex. NeuroImage 13:1002-1015.

Kalatsky VA, Stryker MP (2003) New paradigm for optical imaging: temporally encoded maps of intrinsic signal. Neuron 38:529-545.

Krakow K, Messina D, Lemieux L, Duncan JS, Fish DR (2001) Functional MRI activation of individual interictal epileptiform spikes. NeuroImage 13:502-505.

Kreisman NR, Magee JC, Brizzee BL (1991) Relative hypoperfusion in rat cerebral cortex during recurrent seizures. J Cereb Blood Flow Metab 11: $77-87$.

Kuschinsky W, Paulson O (1992) Capillary circulation in the brain. Cerebrovasc Brain Metab Rev 4:261-286.

Lemieux L, Salek-Haddadi A, Josephs O, Allen P, Toms N, Scott C, Krakow K, Turner R, Fish DR (2001a) Event-related fMRI with simultaneous and continuous EEG: description of the method and initial case report. NeuroImage 14:780-787.

Lemieux L, Krakow K, Fish DR (2001b) Comparison of spike-triggered functional MRI BOLD activation and EEG dipole model localization. NeuroImage 14:1097-1104.

Lindauer U, Royl G, Leithner C, Kuhl M, Gold L, Gethmann J, Kohl-Bareis M, Villringer A, Dirnagl U (2001) No evidence for early decrease in blood oxygenation in rat whisker cortex in response to functional activation. NeuroImage 13:988-1001.

Lindauer U, Gethman J, Kuhl M, Kohl-Bareis M, Villringer A, Dirnagl U (2003) Neuronal activity-induced changes of local cerebral microvascular blood oxygenation in the rat: effect of systemic hyperoxia or pypoxia. Brain Res 975:135-140.

MacVicar BA, Hochman D (1991) Imaging of synaptically evoke intrinsic signals in hippocampal slices. J Neurosci 11:1458-1469.

Malonek D, Grinvald A (1996) Interactions between electrical activity and cortical microcirculation revealed by imaging spectroscopy: implications for functional brain mapping. Science 272:551-554.

Mandeville JB, Marota JJ, Ayata C, Zaharchuk G, Moskowitz MA, Rosen BR, Weisskoff RM (1999a) Evidence of a cerebrovascular postarteriole windkessel with delayed compliance. J Cereb Blood Flow Metab 19:679-689.

Mandeville JB, Marota JJ, Ayata C, Moskowitz MA, Weisskoff RM, Rosen BR (1999b) MRI measurement of the temporal evolution of relative CMRO(2) during rat forepaw stimulation. Magn Res Med 42:944-951.

Masino SA, Kwon MC, Dory Y, Frostig R (1993) Characterization of functional organization within rat barrel cortex using intrinsic signal optical imaging through a thinned skull. Proc Natl Acad Sci USA 90:9998-10002.

Mayhew J, Askew S, Zheng Y, Porrill J, Westby GWM, Redgrave P, Rector DM, Harper RM (1996) Cerebral vasomotion: a $0.1-\mathrm{Hz}$ oscillation in reflected light imaging of neural activity. NeuroImage 4:183-193.

Mayhew J, Johnston D, Berwik J, Jones M, Cofey P, Zheng Y (2000) Spectroscopic analysis of neural activity in brain: increased oxygen consumption following activation of barrel cortex. NeuroImage 12:664-675.
Mehta AD, Suh M, Bahar, Schwartz TH (2003) Optical imaging of epileptiform events in an acute in vivo rat model. Paper presented at 53rd Congress of Neurological Surgeons Annual Meeting, Denver, CO, October.

Nemoto M, Sheth S, Guiou M, Pouratian N, Chen JWY, Toga AW (2004) Functional signal- and paradigm-dependent linear relationships between synaptic activity and hemodynamic responses in rat somatosensory cortex. J Neurosci 24:3850-3861.

Nersesyan H, Hydeer F, Rothman DL, Blumenfeld H (2004) Dynamic fMRI and EEG recording during Spike-Wave seizures and generalized tonicclonic seizures in WAG/Rij rats. J Cereb Blood Flow Metab 24:589-599.

Ogawa S, Lee TM, Kay AR, Tank DW (1990) Brain magnetic resonance imaging with contrast dependent on blood oxygenation. Proc Natl Acad Sci USA 87:9868-9872.

Ojemann GA (1993) Intraoperative tailoring of temporal lobe resections. In: Surgical treatment of the epilepsies (Engel JJ, ed), pp 481-488. New York: Raven.

Pereira de Vasconcelos A, Ferrandon A, Nehlig A (2002) Local cerebral blood flow during lithium-pilocarpine seizures in the developing adult rat: role of coupling between blood flow and metabolism in the genesis of neuronal damage. J Cereb Blood Flow Metab 22:196-205.

Prince DA, Wilder J (1967) Control mechanisms in cortical epileptogenic foci. "Surround" inhibition. Arch Neurol 16:194-202.

Ratzlaff EH, Grinvald A (1991) A tandem-lens epifluorescence macroscope: hundred-fold brightness advantage for wide field imaging. J Neurosci Methods 36:127-137.

Roy C, Sherrington C (1890) On the regulation of the blood supply to the brain. J Physiol (Lond) 11:85-108.

Sato C, Nemoto M, Tamura M (2002) Reassessment of activity-related optical signals in somatosensory cortex by an algorithm with wavelengthdependent path length. Jpn J Physiol 52:301-312.

Schuett S, Bonhoeffer T, Hubener M (2002) Mapping retinotopic structure in mouse visual cortex with optical imaging. J Neurosci 22:6549-6559.

Schwartz EL, Polimeni J, Granquist-Fraser D, Wood RJ (2003) The structure of singular regions in cortical orientation maps: a function of spatial blur. Soc Neurosci Abstr 29:125.4.

Schwartz TH (2003) Optical imaging of epileptiform events in visual cortex in response to patterned photic stimulation. Cereb Cortex 13:1287-1298

Schwartz TH, Bonhoeffer T (2001) In vivo optical mapping of epileptic foci and surround inhibition in ferret cerebral cortex. Nat Med 7:1063-1067.

Schwartz TH, Chen L-M, Friedman RM, Spencer DD, Roe AW (2004) High resolution intraoperative optical imaging of human face cortical topography: a case study. NeuroReport 15:1527-1531.

Schwartzkroin PA, Wyler AR (1980) Mechanism underlying epileptiform burst discharge. Ann Neurol 7:95-107.

Sheth S, Nemoto M, Guiou M, Walker M, Pouratian N, Toga AW (2003) Evaluation of coupling between optical intrinsic signals and neuronal activity in rat somatosensory cortex. NeuroImage 19:884-894.

Sheth SA, Nemoto M, Guiou M, Walker M, Pouratian N, Hageman N, Toga AW (2004) Columnar specificity of microvascular oxygenation and volume responses: implications for functional brain mapping. J Neurosci 24:634-641.

Silva AC, Lee SP, Iadecola C, Kim SG (2000) Early temporal characteristics of cerebral blood flow and deoxyhemoglobin changes during somato sensory stimulation. J Cereb Blood Flow Metab 20:201-206.

Tanaka S, Sako K, Tanaka T, Nishihara I, Yonemasu Y (1990) Uncoupling of local blood flow and metabolism in the hippocampal CA3 kainic acidinduced limbic seizure states. Neuroscience 36:339-348.

Tenney JR, Duong TQ, King JA, Ferris CF (2004) FMRI of brain activation in genetic rat model of absence seizures. Epilepsia 45:576-582.

Vanzetta I, Grinvald A (1999) Increased cortical oxidative metabolism due to sensory stimulation: implications for functional brain imaging. Science 286:1555-1558.

Vanzetta I, Slovin H, Omer DB, Grinvald A (2004) Columnar resolution of blood volume and oximetry functional maps in the behaving monkey: implications for fMRI. Neuron 42:843-854.

Vnek N, Ramsden BM, Hung CP, Goldman-Rakic PS, Roe AW (1999) Optical imaging of functional domains in the cortex of the awake and behaving monkey. Proc Natl Acad Sci USA 96:4057-4060.

Wyllie E, Lüders H, Morris HH (1987) Clinical outcome after complete or partial cortical resection for intractable epilepsy. Neurology 37 : $1634-1641$. 